\title{
Mise À Jour De La Cartographie Géologique Et États Des Contraintes Tectoniques Dans La Zone De Bouchfâa (NW Du Massif De Tazekka, Maroc)
}

\author{
Youssef Gharmane, \\ Mohamed El Fartati, \\ Samir Amrani, \\ Saïd Hinaje,
}

Laboratoire de Géo-ressources et Environnement, Université Sidi Mohamed Ben Abdellah, Faculté des Sciences et Techniques, Fès, Maroc

Doi: 10.19044/esj.2018.v14n18p193 URL:http://dx.doi.org/10.19044/esj.2018.v14n18p193

\begin{abstract}
The tectonic and microtectonic analysis of the Paleozoic and MesoCenozoic series of the Bouchfâa zone (NW of Tazekka) enabled us to highlight tectonic palaeo-stress of essentially compressive stresses. These played a key role in the uprising of the Tazekka Massif. The age and chronology of these paleo-stress are established by analogy with the results obtained in the Middle Atlas, Saïs and High Moulouya. The N165 major compressive phase of post-Bathonian and ante-Barremianage, responsible for the thrust of Lower Lias deposits with those of Middle Lias. The second major compressive episode is oriented N120 in the Vallesian and N140 in the lower Pliocene. These last two tectonic events are responsible for the formation of a ramp-flat system and strik-slep thrust oriented N20 to N70. They affect the paleozoic base of the Tazekka massif and its cover.
\end{abstract}

Keywords: Tectonic, Ramp-Flat, Middle Atlas, Tazekka, Morocco

\section{Résumé}

Les analyses tectoniques et microtectoniques des séries paléozoïques et méso-cénozoïques de la zone de Bouchfâa (NW de Tazekka), nous ont permis de mettre en évidence des paléo-champs de contraintes essentiellement compressifs. Ceux-ci ont joué un rôle primordial dans le soulèvement du massif de Tazekka. L'âge et la chronologie de ces paléo-contraintes sont établis par analogie avec les résultats obtenus au Moyen Atlas, Saïs et la Haute Moulouya. La phase compressive majeure orientée N165 d'âge postBathonien et anté-Barrémien, responsable du chevauchement des dépôts du 
Lias inférieur sur ceux du Lias moyen. Le deuxième épisode compressif majeur est orienté N120, d'âge Vallésien. Le dernier événement compressif majeur est orienté N140 au Pliocène inférieur. Ces deux derniers événements tectoniques sont responsables de la formation d'un système palier-rampe de chevauchements et décro-chevauchements de direction N20 à N70. Ils affectent le socle paléozoïque du massif de Tazekka et sa couverture.

Mots-clés: Tectoniques, palier-rampe, Moyen Atlas, Tazekka, Maroc

\section{Introduction}

Le secteur qui fait l'objet de cette étude appartient à une zone de transition entre le couloir sud Rifain, le causse moyen atlasique et le massif de Tazekka. Cette zone est limitée au Nord et à l'Ouest par la zone effondrée sud rifaine, et vers l'Est et le Sud par le causse moyen atlasique et le massif hercynien de Tazekka (figure 1. B).

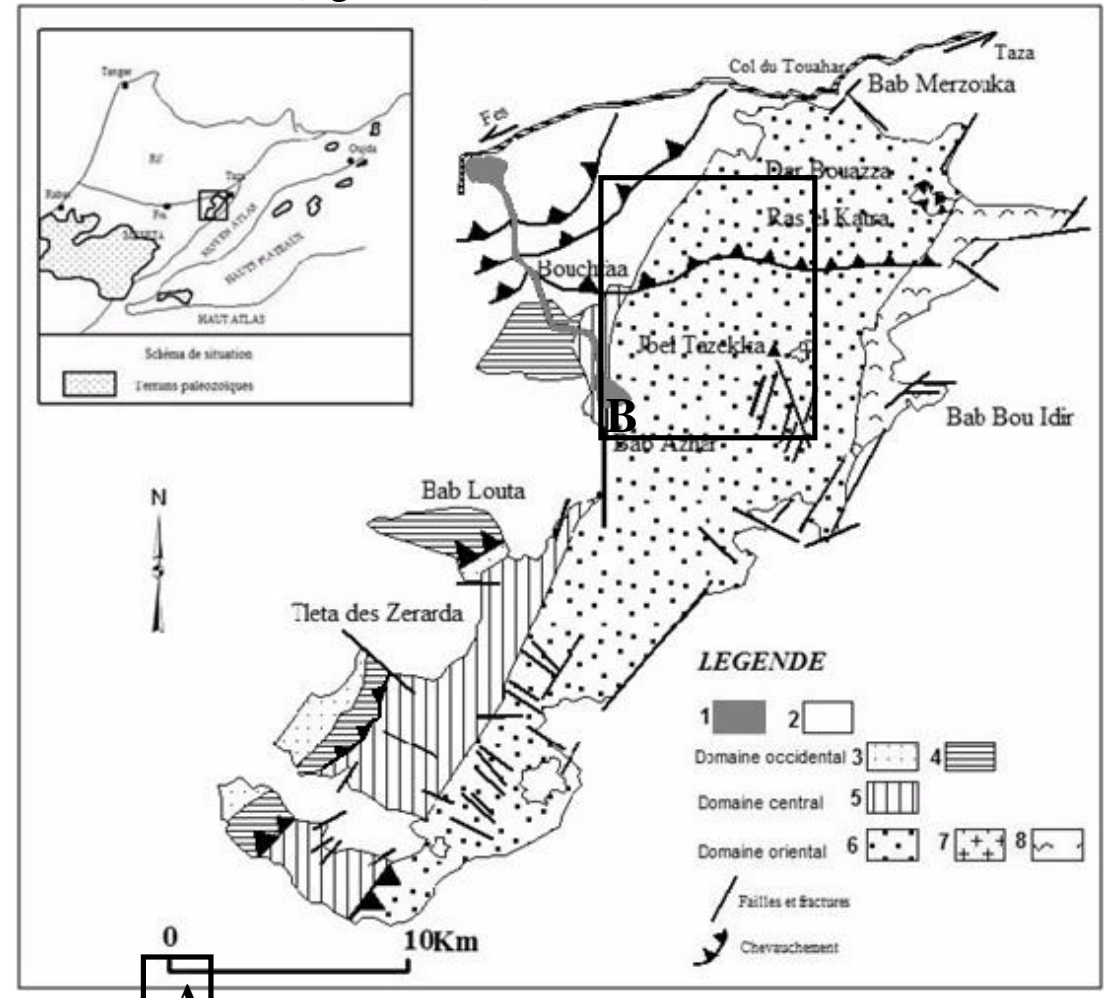

Figure 1 : A-Aarte géologique schématique de la boutonnière de Tazekka (d'après Hoepffner, 1978 ; modifiée). B- Localisation de la zone étudiée.

1-Volcanisme quaternaire ; 2-Couverture post-hercynienne ; 3-Formations détritiques : Ordovicien inférieur et Silurien-Dévonien ; 4-Quartzites et schistes: Ordovicien supérieur ; 5- Schistes vert olive à niveau quartzitiques ; Ordovicien inférieur ; 6-Schistes de Tazekka :

Cambro-Ordovicien ; 7-Granite du Tazekka ; 8-Complexe volcano-sédimentaire : Viséen supérieur-Namurien. 


\section{Objectif du travail}

Notre travail consiste la mise à jour de la partie NE de la carte géologique 1/50000 de Tahla (Vidal. J et C. Hoepffner, 1979) (Fig. 2). Celleci montre l'intercalation des argiles rouges attribuées au Domérien, au milieu des couches de calcaires oolitiques en gros bancs d'âge domérien. Ces niveaux argileux correspondent en réalité à des surfaces de chevauchement striés, ramenant des niveaux argileux triasiques. Le deuxième objectif est de mettre en évidence les phases tectoniques qui ont structuré le massif de Tazekka et la zone de passage vers le couloir sud rifain, et d'essayer d'expliquer le soulèvement du socle paléozoïque de ce Massif.
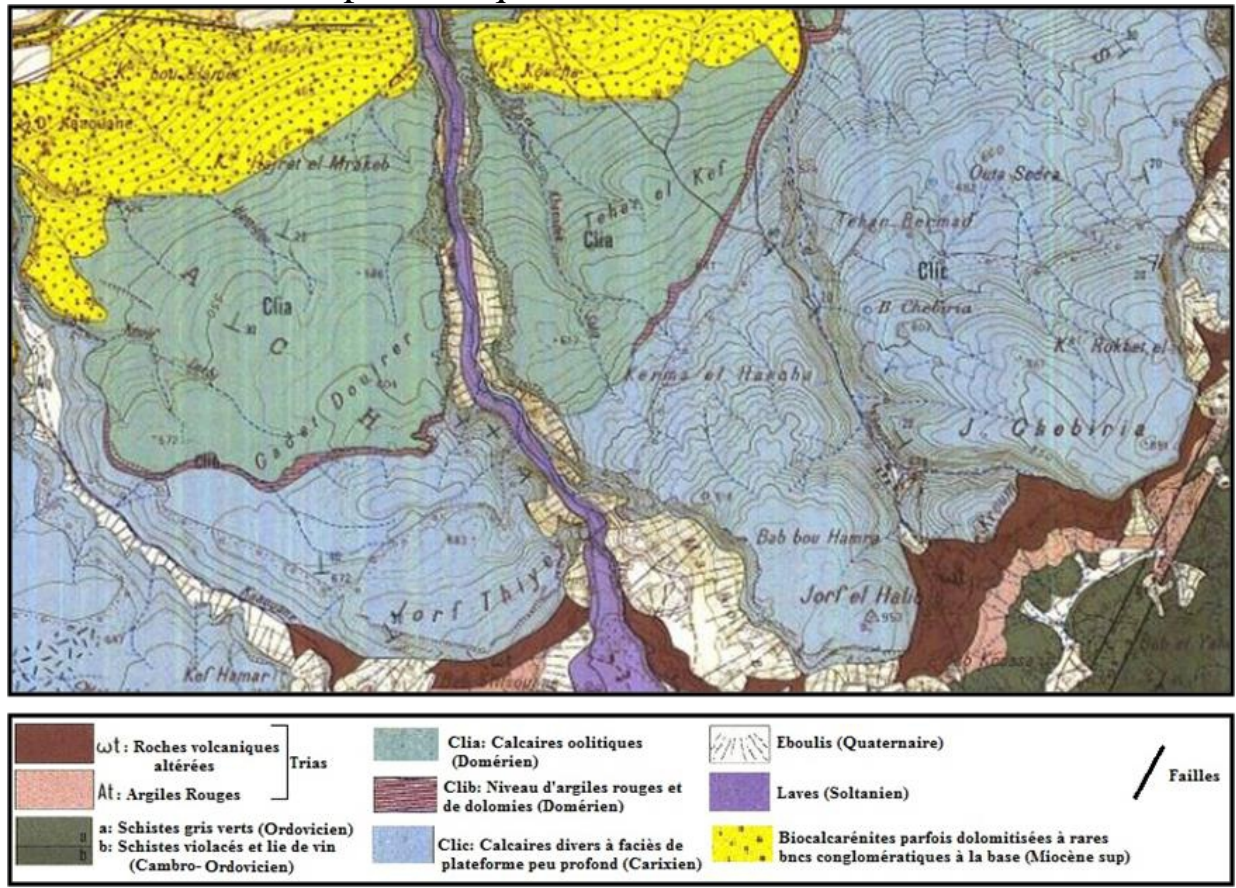

Figure 2 : carte géologique 1/50000 de la zone de Bouchfâa au NW de la boutonnière de

Tazekka (d'après Vidal. J et C. Hoepffner, 1978).

\section{Aperçus stratigraphique et lithologique de la zone d'étude}

Les séries sédimentaires paléozoïques et méso-cénozoïques, affleurant dans la zone d'étude, correspondent successivement aux formations suivantes :

- les formations cambro-ordoviciennes et ordoviciennes inférieur (Arénigien-Llanvirnien), constituées essentiellement d'un faciès schisteux violacé (lie de vin) ou gris-vert (Hoepffner, 1987 ; Rauscher et al, 1982), comprenant des argilites schisteuses à minces lits de grés micacés, des grésopélites à dominance de pélites et de silts, et des grès grauwackeux. Ces dépôts sont interprétés comme une méga-séquence strato et granocroissante de type plateforme subsidente (Ajamay, 2010); 
- $\quad$ ces formations schisteuses sont surmontées en discordance par des faciès triasiques comprenant deux séries d'argilites rouges séparées par un complexe basaltique doléritique (Manspeizer et al, 1978 ; Robillard, 1978, 1979, 1981 ; Benchekroun, 1985) ;

- $\quad$ les dépôts liasiques formés successivement de dolomies bréchiformes, fines et grises à structures du type Birdeyes d'âge Lotharingien, des calcaires divers à faciès de plateforme peu profonde qui marque le début du Carixien (Robillard, 1981) et des calcaires oolitiques en gros bancs d'âge Domérien (Vidal J. et C. Hoepffner, 1979 ; Robillard, 1978) ;

- les dépôts miocènes discordants, constitués de grés et des marnocalcaires à caractère transgressif (Cirac, 1985), recouverts par une formation fluvio-lacustre d'âge Pliocène supérieur où se côtoient sables, calcaires et calcilutites (Dridri et Fedan, 2001) ;

- les dépôts quaternaires caractérisés par des formations volcaniques sous forme de cendres, tufs, brèches volcaniques et laves, travertins et dépôts fluviatiles.

\section{Analyses tectonique et cartographique}

A l'échelle cartographique ; les observations et mesures de terrain ainsi que l'analyse de la carte géologique 1/50000 et des images satellitaires ; nous ont permis d'aboutir à une classification des directions des fractures prédominantes. Ainsi nous avons pu distinguer trois directions principales de failles transversales et longitudinales. Les différentes familles de failles sont : - $\quad$ N50 à N80 à jeux inverse et chevauchant. Elles sont responsables de l'intercalation des argiles rouges triasiques au milieu des couches domériennes sous forme d'écailles tectoniques surmontant les dépôts du Lias inférieur. Ce contact a été interprété comme un contact stratigraphique normal, avec des argiles rouges attribués au Domérien (Vidal J. et C. Hoepffner, 1979) (voir Fig. 2). Ces failles se prolongent latéralement jusqu'aux socles schisteux ordovicien situé au NW de la boutonnière paléozoïque de Tazekka. Elles affectent localement les dépôts du miocène supérieur (n5-6: Ramirez Merino et al, 2008) au Sud du couloir sud Rifain ;

- $\quad$ N30 à N40 à jeux inverse et chevauchant responsables de la formation d'un système d'écailles tectoniques par découpage en baïonnette métrique des schistes gris-verts, grés micacés, pélites grises et quartzites. Cette structure correspond à un système en palier-rampe de chevauchements et décrochevauchements en duplex, dont les anticlinaux des rampes sont entièrement érodés. Cette famille présent aussi un rejeu tardif en décrochement senestre ; - $\quad$ N160 à N00 présentent des jeux normaux et décalent les fractures précédentes. Ces failles affectent même les coulées et les épanchements volcaniques récents. Ces derniers recouvrent une terrasse fluviatile du Pléistocène supérieur (Dridri et Fedan, 2001). 
A l'échelle de l'affleurement; les formations liasiques montrent un passage vertical qui se fait par contact chevauchant avec duplication des couches triasico-liasiques. Ces écailles tectoniques métriques, sont délimité par des failles orientées N50 à N80, à pendages variant de 25 à $60^{\circ}$ vers le NW et le NNW. Les argiles rouges et basaltes altérés triasiques forment les principaux niveaux de décollement correspondant à des paliers de chevauchements. Près de Douar Et-Tsine, au sein du faciès schistes lie de vin et schistes gris-vert d'âge successivement Cambro-ordovicien et Ordovicien inférieur (Arénigien-Llanvirnien); les fractures N50 à N80 sont à remplissage quartzeux riche en oxydes et hydroxydes de fer. Les chevauchements avec duplicatures affectant les schistes ordoviciens et montrant des lambeaux du socle, sont accompagnés d'affleurements triasiques argileux et basaltiques. Ceci montre que ces chevauchements attribués à tort aux phases hercyniennes, possèdent au moins un rejeu d'âge atlasique.

\section{Analyse microtectonique et état des contraintes}

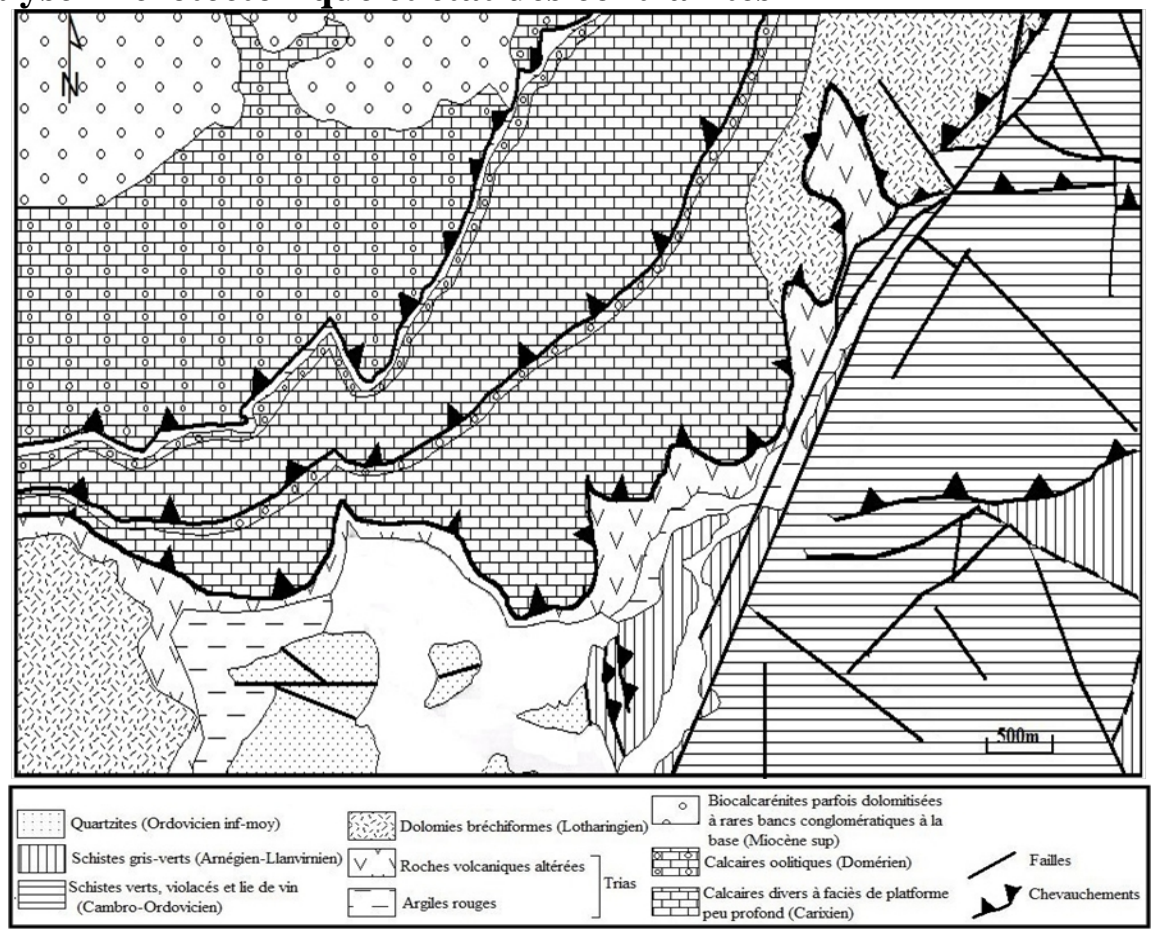

Figure 3 : Carte géologique schématique de la région de Bouchfâa située au NW de la boutonnière de Tazekka.

Les mesures microtectoniques de la déformation cassante effectuées au sein des couches d'âge paléozoïque, liasique, et quaternaire, ainsi que les levés cartographique (Fig. 3) et les coupes structurales; nous ont permis de décrire un système tectonique en pallier-rampe sur chevauchements N80 à 
N50 (Fig. 4). Ces failles affectent à la fois le socle paléozoïque et les épaisses formations liasiques liées à la subsidence synsédimentaire (Robillard, 1978). L'érosion intense a provoqué la disparition de tout l'anticlinale de rampe. Ce système est contrôlé par des failles chevauchantes et des failles inverses orientées N50 à N80, résultant d'une compression sub-méridienne. Nos analyses microtectoniques et le traitement automatique des mesures, nous ont permis de mettre en évidence ce paléochamp de contraintes qui est caractérisé par une contrainte maximale $\sigma_{1}$ horizontale et orientée N165 associée à une contrainte minimale $\sigma_{3}$ verticale (Fig. 7, A). Ce paléochamp de contraintes est d'âge post-Bathonien et anté-Barrémien (Hinaje, 2004). Le rapport de forme $\phi=(\sigma 2-\sigma 3) /(\sigma 1-\sigma 3)=0.30$; indique que les failles inverses et les failles chevauchantes peuvent avoir une composante décrochante.

Le jeu de ces failles a provoqué la formation des écailles tectoniques matérialisées par des duplications des bancs de calcaire oolitique d'âge Domérien (Ph. 1). Les surfaces en paliers correspondent aux niveaux argileux rouges d'âge triasique (couches savons). Nous avons pu dénombrer quatre plans de failles chevauchantes majeures associées à des dizaines de plans mineurs de failles inverses. Ces plans délimitent des écailles tectoniques enracinées vers le NW, et affectant le sommet des argiles rouges triasiques, le sommet et le milieu des calcaires oolitiques carixiens. Ces failles présentent un rejeu décrochant dextre à composante inverse, résultant d'une compression N125. Ce jeu est compatible avec un paléochamp de contraintes tel que l'axe de la contrainte principale $\sigma_{1}$ est horizontal et orienté N125 et l'axe $\sigma_{3}$ est subhorizontal (Fig. 7, B). Cette compression est d'âge Vallésien et probablement avec un rejeu d'âge Pliocène inférieur (Hinaje 2004). Le rapport $\phi$ varie de 0,11 à 0.45 indiquant que les dérochements peuvent être associés à des failles inverses et/ou présentent une composante verticale inverse. Ces jeux que nous venant de décrire, affectent également les couches carbonifères du massif de Tazekka.

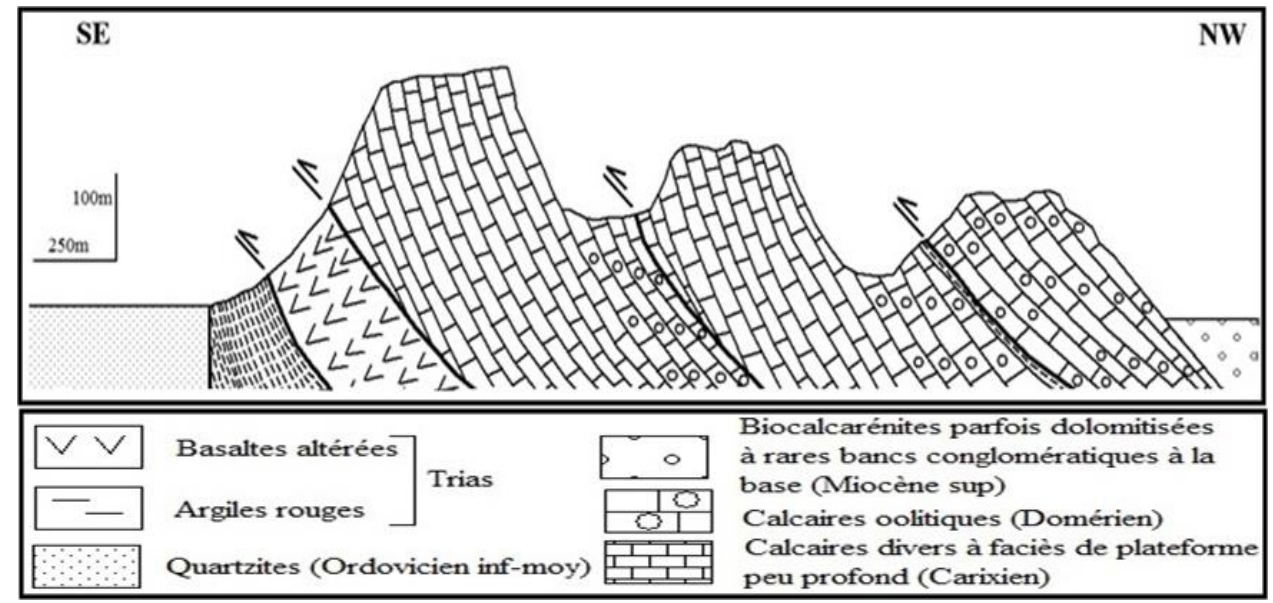

Figure 4 : Coupe géologique au SW du Massif de Tazekka. 


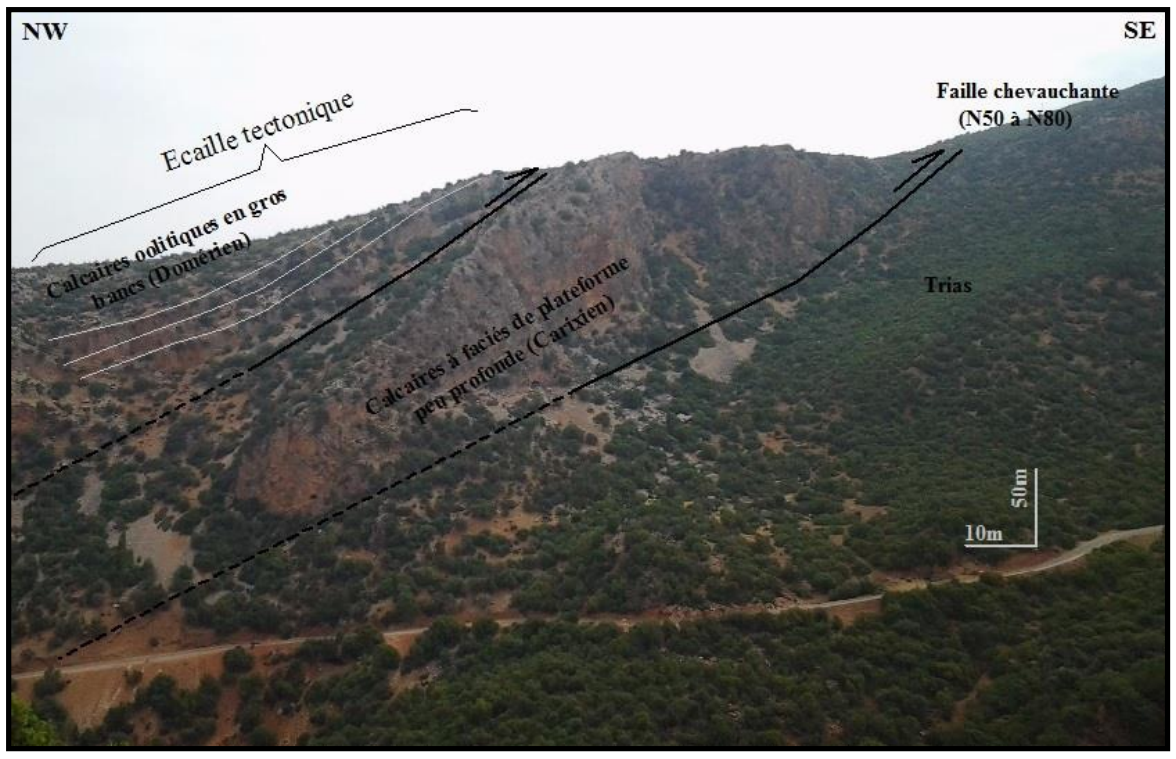

Photo 1 : Tectonique en palier-rampe dans la zone de Bouchfâa.

Près de Douar Et-Tsine, l'ensemble des failles mesurées sur le terrain sont orientées en générale N50 à N80. Elles affectent les schistes lie de vin et les schistes gris-verts d'âge successivement Cambro-Ordovicien et Ordovicien inférieur (Arénigien-Llanvirnien). Dans ces couches nous avons pu mesurer des failles chevauchantes présentant deux jeux. La chronologie des jeux de failles est basée sur la superposition des stries sur les plans de failles, les recoupements des plans et l'âge des formations. Les âges des phases et épisodes tectoniques sont établis par analogie avec les résultats obtenus au Moyen Atlas, Saïs et la Haute Moulouya par S.Hinaje (2004). Le jeu des failles N50 à N80 est compatible avec un paléochamp de contraintes caractérisé par un régime compressif tel que l'axe de la contrainte principale $\sigma_{1}$ est horizontal et orienté $\mathrm{N} 25\left(\sigma_{3}\right.$ verticale) (Fig. $\left.7, \mathrm{C}\right)$. Cet état de contraintes est à l'origine du jeu décrochant senestre des failles orientées N50 à N80 avec parfois une composante inverse. Il est aussi responsable de l'installation d'un système filonien sur failles N50 à N80 dont la striation et synchrone avec les minéralisations quartzeuses à oxydes et hydroxydes de fer de couleur brune à rouge. L'âge de cette phase tectonique est attribué au Paléogène-Miocène inférieur.

Le système filonien sur failles N50 à N80, a enregistré une déformation post-minéralisation quartzeuse. Celle-ci est matérialisée par des stries affectant les épontes des lentilles minéralisées. Le paléochamp de contraintes responsable de ce jeu est caractérisé par un régime compressif orienté N100 $\left(\sigma_{1}\right.$ horizontale et orientée N100, $\sigma_{3}$ verticale) (Fig. 7, D), responsable du jeu décrochant dextre des failles orientées N70 à N80. Puisque ces failles affectent à la fois le socle paléozoïque et la couverture triasico-jurassique, nous 
attribuons à cette phase tectonique un âge Vallésien ; par analogie avec les résultats obtenus au Moyen Atlas (Hinaje, 2004).

Les failles orientées N50 à N80, présentent des jeux multiple dont le plus important correspond au jeu chevauchant. Ce jeu remobilise des lambeaux de schiste lie de vin avec injections des argiles et basaltes triasiques. Il faut signaler également le chevauchement de lambeaux triasico-jurassiques sur les schistes violacés et lie de vin (Fig. 5). Par endroits nous avons noté des fentes plates métriques de quartz ferrifères, témoignant d'un axe $\sigma_{3}$ vertical dont l'intensité est inférieur à la pression des fluides (Fig. 6). Les surfaces de chevauchements épousant parfois les lits de pélites stratifiées d'âge CambroOrdovicien et à pendage variant de 0 à $40^{\circ} \mathrm{NW}$; forment des surfaces en paliers (Graham et al. 1987). Les couches rebroussées des schistes violacés et lie de vin sont scellées par des éboulis et des cônes de déjection récents au Nord du Douar Boughoufa.

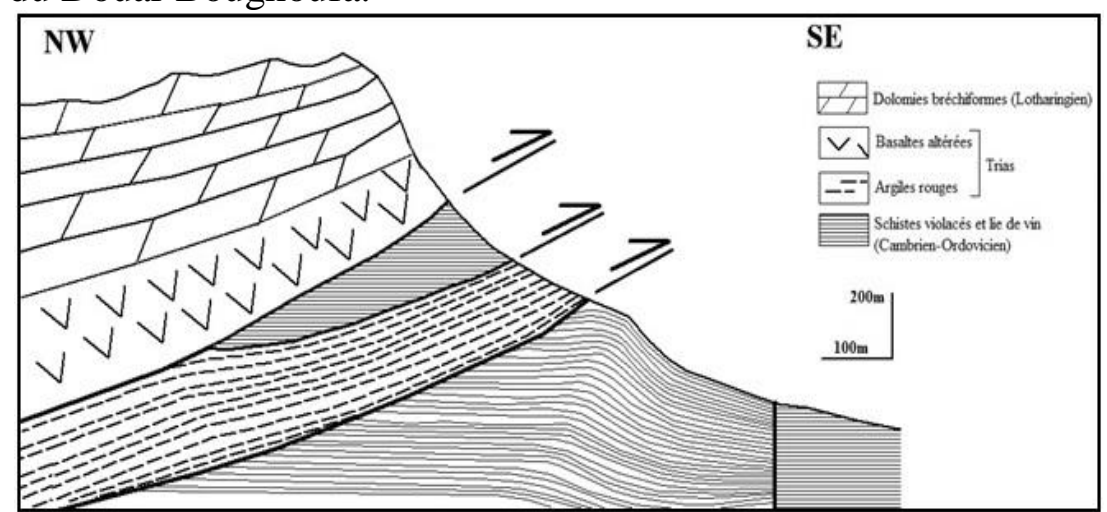

Figure 5 : Coupe géologique montrant le chevauchement de lambeaux Triasico-Jurassique sur les schistes violacés à Douar Et-Tsine.

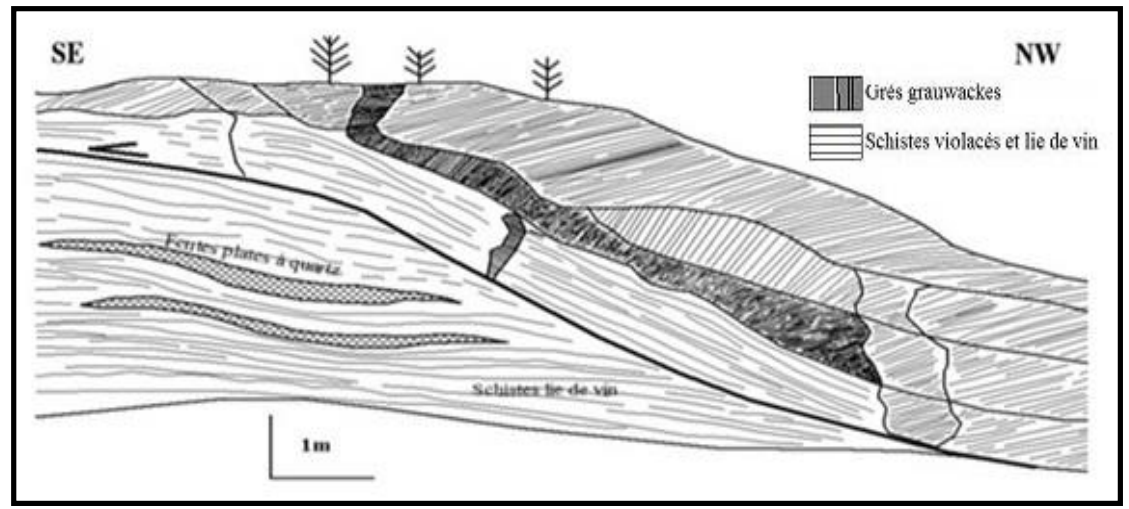

Figure 6 : Chevauchement en palier et fentes plates de quartz à oxydes et hydroxydes de fer au Nord de Douar Et-Tsine.

Les coulées volcaniques récentes, situées au niveau des vallées, sont affectées par des failles normales orientées N160 à N00. Ces failles sont le résultat de l'extension N60 à N80, tels que l'axe de la contrainte principale $\sigma_{1}$ 
est vertical, et l'axe de la contrainte minimale $\sigma_{3}$ est horizontal et orienté N60 à N80 (Fig. 8, E), dont l'âge est attribué au Quaternaire moyen-récent (Hinaje, 2004). Le rapport de forme $\phi=0.15$ montre que les valeurs de $\sigma_{2}$ et $\sigma_{3}$ sont très proches. Les failles normales sub-méridiennes présentent parfois une composante décrochante senestre.

Nous avons procédé par principe d'élimination des phases tectoniques récentes affectant les terrains anciens. Ainsi, les analyses microtectoniques que nous avons effectuées dans le socle paléozoïque de Tazekka, nous ont permis de retrouver les mêmes phases tectoniques que nous avons décrites dans la couverture triasico-liasique et même quaternaire. Nous avons mis en évidence des failles décrochantes dextres orientées N75 à N100, affectent les couches de grés micacés et pélites grises d'âge Ordovicien supérieur. Ces failles sont le résultat de la compression N110, tels que l'axe de la contrainte maximal $\sigma_{1}$ est orienté $\mathrm{N} 110$ avec un plongement de $28^{\circ} \mathrm{WNW}$, l'axe minimal $\sigma_{3}$ est orienté $\mathrm{N} 40$ avec un plongement de $30^{\circ} \mathrm{NE}$ et l'axe intermédiaire $\sigma_{2}$ est orienté N168 avec un plongement de $45^{\circ}$ SSE (Fig. 8, F). Puisque nous avons trouvé le même état de contraintes dans la couverture ; cette phase tectonique peut être d'âge hercynien et/ou d'âge Vallésien voire même Pliocène inférieur.

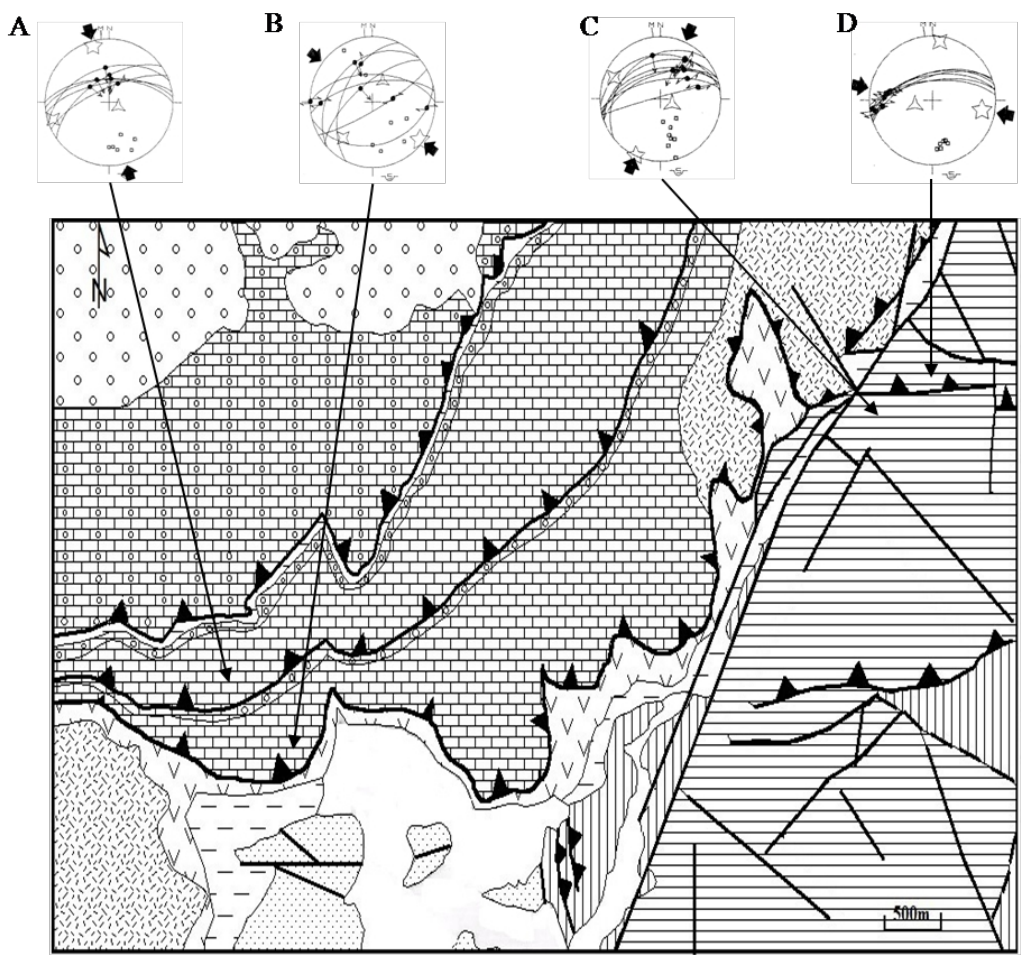

Figure 7 : Carte géologique schématique de la région de Bouchfâa et stéréogrammes des épisodes tectoniques équivalents. (Flèche centrifuge : extension; flèche centripète : compression; étoiles à 3,4 et 5 branches représentent respectivement les contraintes $\sigma_{3}, \sigma_{2}$ et $\sigma_{1} ; \mathrm{MN}$ : Nord magnétique et Nord géographique (diagramme de schmidt, hémisphère inférieur)). 


\section{Activité volcanique}

L'activité volcanique récente a été décrite dans le Moyen Atlas et la Haute Moulouya, comme liée à des fentes de tension sub-méridiennes dans un système de décrochements senestres NE-SW (Harmand et Cantagrel, 1984 ; Harmond et Moukadiri, 1986 ; El Azouzi et al, 1999). Puisque l'âge du volcanisme s'étale du Néogène jusqu'au Quaternaire récent; donc ce volcanisme il est associé à plusieurs phases et épisodes tectoniques avec des géométries d'ouverture variables (Charrière, 1990 ; Hinaje et Aït Brahim, 2002 ; Hinaje, 2004). Dans notre zone d'étude, cette activité volcanique est probablement liée à des nœuds tectoniques qui fonctionnent comme des zones de relais en transtension. Ces zones correspondent à l'intersection des failles normales N160 à N00, et les failles décrochantes dextres N80 à N90 (Fig. 8).

Près de Souq Sabt, nous avons mesuré des failles décrochantes senestres orientées N30 à N60, affectant à la fois les couches de grés micacés (Ordovicien supérieur), les pélites grises (Ordovicien supérieur), les schistes gris-verts (Arénigien-Llanvirnien), les schistes violacés et lie de vin (Cambroordovicien), ainsi que les coulés basaltiques quaternaires (Soltanien). Ces failles sont le résultat d'une compression sub-méridienne, tels que l'axe de la contrainte maximal $\sigma_{1}$ est horizontal et orienté N170, et l'axe de la contrainte minimal $\sigma_{3}$ est aussi horizontal et orienté N80 (Fig. 8, G). Nous attribuons à cette phase tectonique un âge Quaternaire moyen-récent, puisqu'elle affecte les coulées basaltiques. Le rapport de forme $\phi=0.55$, indique que cet état de contraintes correspond à un épisode de la phase tectonique d'âge Quaternaire. Un premier épisode de cette phase, a donné naissance à des failles normales sub-méridiennes probablement synchrones avec le volcanisme récent. Ces failles sont légèrement décalées par des décrochements senestres N30 à N60, lors du deuxième épisode tectonique de cette phase récente. 


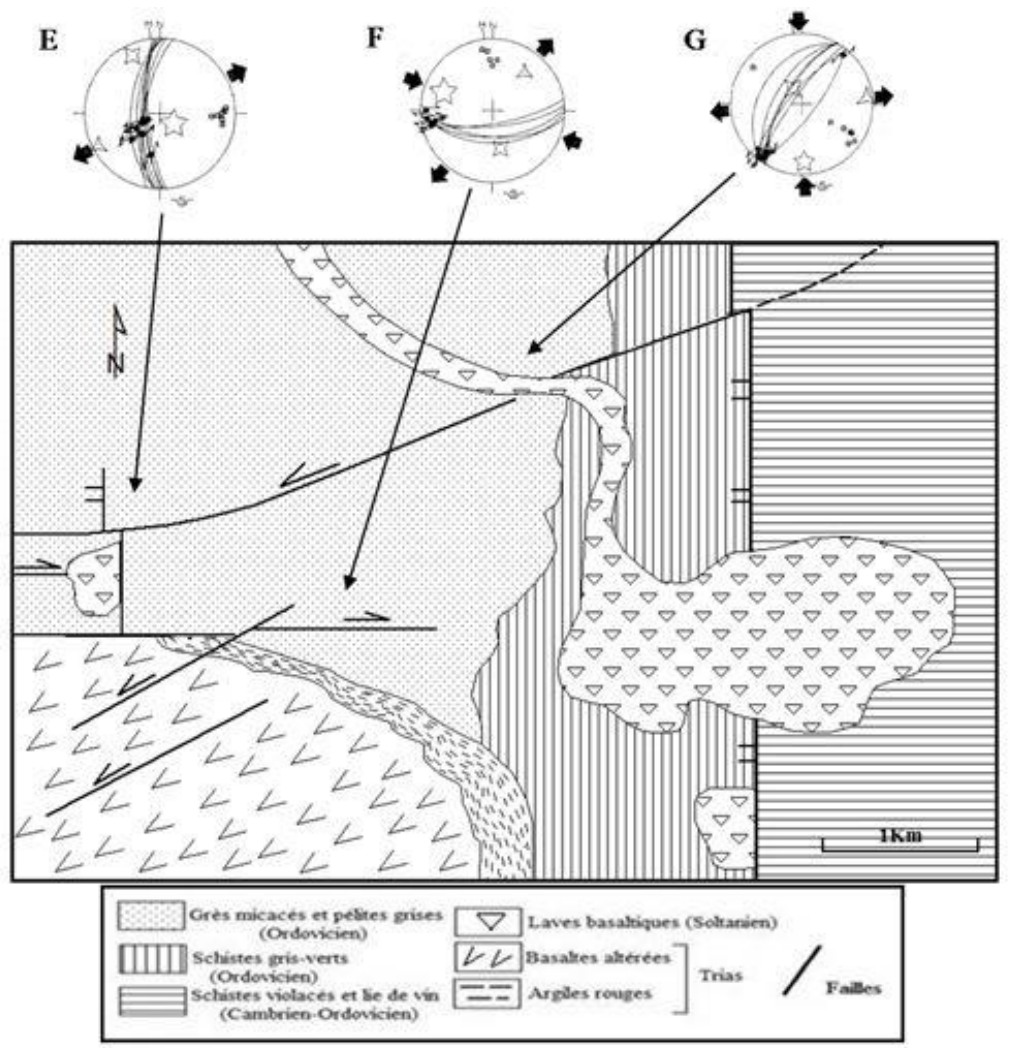

Figure 8 : Schéma structural des épanchements basaltiques quaternaires de Koudiat Bab Douma et stéréogrammes des épisodes tectoniques équivalents. (Flèche centrifuge : extension ; flèche centripète : compression ; étoiles à 3,4 et 5 branches représentent respectivement les contraintes $\sigma_{3}, \sigma_{2}$ et $\sigma_{1} ; \mathrm{MN}$ : Nord magnétique et Nord géographique (diagramme de schmidt, hémisphère inférieur)).

\section{Conclusion}

La structure complexe de la zone étudiée, située à cheval entre le massif paléozoïque de Tazekka, le Moyen Atlas et le couloir sud rifain ; résulte d'une évolution tectonique polyphasée. Au post-Bathonien-anté-Barrémien, le paléochamp de contraintes est caractérisé par un axe $\sigma_{1}$ horizontal orienté N160. Les états de contraintes caractérisés par des compressions N30-N40, N110-N120 et N130-N140, sont attribués au Paléogène-Miocène inférieur, au Vallésien et au Pliocène inférieur. Au Quaternaire moyen-récent, le régime tectonique est matérialisé par des failles normales N160 à N00, et des décrochements senestres N30 à N60. Ces phases et épisodes tectoniques d'âge atlasique, sont superposés avec les mêmes directions des contraintes des phases hercyniennes. Ceci rend l'étude de la fracturation plus difficile dans le socle paléozoïque. Pour cela nous avons procédé par le principe des éliminations des phases tectoniques depuis les terrains les plus récents 
(Quaternaire) vers les terrains les plus anciens (Cambro-ordovicien et Ordovicien inférieur (Arénigien-Llanvirnien)).

\section{References:}

1. Ajamy, H. (2010). Evolution tectono-métamorphique du NW du Massif de Tazekka: implication métallogénique. Thèse $3^{\circ}$ cycle, Rabat, 156p.

2. Benchekroun, M.S. (1985). Les séries triasique du Maroc (domaine mésétien et atlasique): évolution minéralogique et géochimique. Thèse $3^{\circ}$ cycle, Univ. Strasbourg, 129p.

3. Cirac, P. (1985). Le bassin sud-rifain occidental au Néogène supérieur. Evolution de la dynamique sédimentaire et de la paléogéographie au cours d'une phase de comblement. Thèse ès-Sciences, Univ. Bordeaux I, France, 2 t, 285 p.

4. El azzouzi, M., Griffiths, B., Bellon, H., Maury, R. C., Piqué, A., Fourcades., Cotten, J., et Hernandez, J. (1999). Evolution des sources du volcanisme marocain au cours du Néogène. C. R. Acad. Sci. Paris, 329, pp. 95-102.

5. Charrière, A. (1990). Héritage hercynien et évolution géodynamique alpine d'une chaîne intracontinentale : le Moyen Atlas au SE de Fès (Maroc). Thèse Doc. Etat, Toulouse, 589p.

6. Graham, R., Hossack, J., Deramond, J., Soula, J.C. (1987). Géométrie des surfaces de chevauchement. Bull. Soc. Géol. France, (8), t. III, n 1 , pp. 169-181.

7. Dridri, A., et Fedan, B. (2001). Rôle du contrôle structural dans la mise en place du réseau hydrographique de Sebou et d'Inaouène entre Fès et oued Amlil (Maroc). Bull. Inst. Sci. Rabat, section Sciences de la Terre, $\mathrm{n}^{\circ} 23,67-77$.

8. Hinaje, S., et Aït Brahim, L. (2002). La chaîne atlasique marocaine : résultat du rifting triasico-liasique et d'une tectonique polyphasée méso-cénozoïque. Sixth International Conférence on the Geology of the Arab World (GAW-6) (Cairo Univ, Gisa, Egypt).

9. Hinaje, S., et Aït Brahim, L. (2002). Paléocontraintes triasico-liasique dans le Moyen Atlas: modèle d'ouverture et fermeture du rifting atlasique NE-SW. $3^{\circ}$ Réunion du Groupe Marocain du Permien et du Trias (GMPT3).

10. Hinaje, S. (2004). Tectonique cassante et paléochamps de contraintes dans le Moyen Atlas et le Haut Atlas central (Midelt-Errachidia) depuis le Trias jusqu'à l'actuel. Thèse Doc. Etat, Univ. Rabat, 363p.

11. Hoepffner, C. (1978). Le massif paléozoïque du Tazekka (Maroc) ; analyse des déformations liées à un linéament tectonique. Bull. Sci. Géol, Strasbourg, 31, 1, pp. 33-44. 
12. Hoepffner, C. (1987). La tectonique hercynienne dans l'Est du Maroc. Thèse Doc. Etat,Univ. Strasbourg, 276p.

13. Harmond, C., et Cantagrel, J.M. (1984). Le volcanisme alcalin tertiaire et quaternaire du Moyen Atlas (Maroc) : chronologie K/Ar et cadre géodynamique. J. Afric. Earth. Sc. Vol. 2, n 1, pp. 595-603.

14. Harmond. C., et Moukadiri A. (1986). Synchronisme entre tectonique compressive et volcanisme alcalin: exemple de la province quaternaire du Moyen Atlas (Maroc). Bull. Soc. Géol. France, (8), t. II, $n^{\circ} 4$, p. 595-603.

15. Manspeizer, W., Puffer, J.H., cousminer H.L. (1978). Separation of Morocco and easternnorthAmerica: A Triassic-Liassicstratigraphic record. Geol. Soc. America Bull., 89, 901-920.

16. Robillard, D. (1978). Etude stratigraphique et structurale du Moyen Atlas Septentrional (Région de Taza-Maroc). Thèse $3^{\circ}$ cycle, Lille, 178p.

17. Robillard, D. (1979). Tectonique synsédimentaire du Moyen-Atlas septentrional au sud de Taza (Maroc). Bull. Soc. Géol. France, 1979, (7), I. XXI, nº 4, p. 441-447.

18. Robillard, D. (1981). Etude stratigraphique et structurale du Moyen Atlas septentrional (région de Taza, Maroc). Notes du Serv. Géol. Maroc. 42 (308), 101-193.

19. Ramirez Merino, J.I., Portero, G., Aït Brahim, L., Chalouan, A., Hoepffner, C., Tahiri El ousrouti, M. (2008). Carte géologique du Maroc 1/50000, feuille de Taza. Notes et mémoires Serv. Géol. Maroc, $\mathrm{n}^{\circ} 433$ bis.

20. Raucher, R., Marhoumi, R., Vanguestaine, M., Hoepffner, C. (1982). Datation palynologique des schistes du Tazekka au Maroc. Hypothèse structurale sur le socle hercynien de la Meseta oriental. C.R. Acad. Sci. Paris, 294 (D), 1203-1206.

21. Vidal, J., et C, Hoepffner. (1979). Carte géologique au 1/50000, feuille de Tahla. Notes et Mémoires Serv. Géol. Maroc, n²282. 